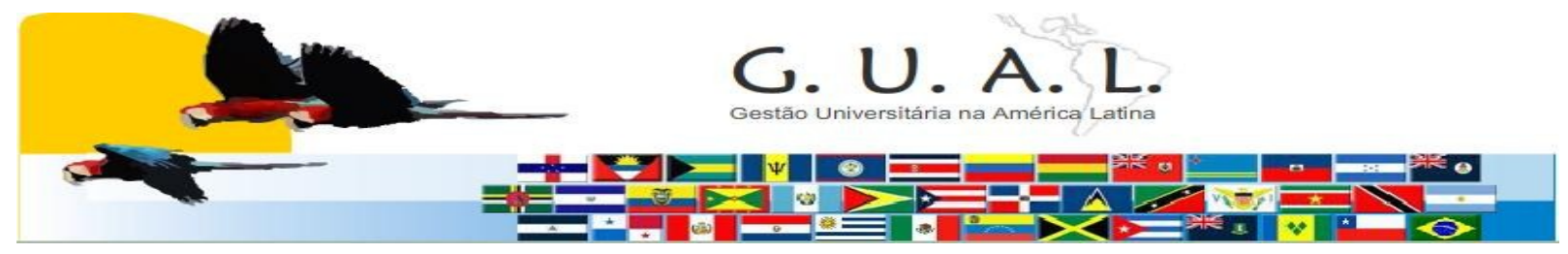

ISSN 1983-4535

\title{
REALINEAMIENTO UNIVERSITARIO EN LA ISLA DE PUERTO RICO
}

\author{
Rafael Aragunde, Doutor \\ Universidad Interamericana de Puerto Rico \\ aragunde@yahoo.com
}

\section{RESÚMEN}

La dinámica universitaria en Puerto Rico atraviesa por una situación muy especial. La Universidad de Puerto Rico, que es la universidad pública del gobierno, ha sufrido de paros y huelgas en estos últimos dos años debido a un aumento de matrícula impuesto en contra de la voluntad de los estudiantes. Mientras tanto, el Gobierno ha nombrado un grupo de trabajo de ciudadanos identificados con políticas neo liberales para que prepare un proyecto de reforma universitaria que se espera que elimine instancias de participación estudiantiles y profesorales que habían contribuido a democratizar la institución. La historia de la educación en Puerto Rico, desde la llamada conquista de los españoles, pasando por la invasión de los Estados Unidos en el 1898, y hasta llegar a estos comienzos del siglo veintiuno con su impactante recesión económica deben ser repasadas para entender cómo el país ha tenido que luchar para alcanzar los porcentajes altísimos de actividad académica, tanto escolar como universitaria, que le caracterizan y que se podría trastocar con la situación que se ha creado. En tal coyuntura, sin embargo, el sector universitario privado, que ya atiende más del $70 \%$ de los estudiantes, tendrá una gran oportunidad de desarrollarse. Si opta por respaldar la investigación científica y no se limita a la enseñanza podrá contribuir generosamente a un realineamiento universitario que cambiará la personalidad de la educación superior puertorriqueña.

Palabras claves: Realineamiento. Puerto Rico. Universidad. 


\section{Realineamiento Universitario En La Isla De Puerto Rico}

En estos precisos instantes la dinámica universitaria puertorriqueña atraviesa por uno de los momentos de mayor incertidumbre en su historia. Mucho está en juego y poco se sabe sobre las intenciones que el partido de gobierno tiene para con una institución creada en el 1903 y que le ha servido generosamente al país caribeño que es Puerto Rico. A la vez, el número de estudiantes del sector de las IES privadas del país crece abruptamente y ante la situación de inestabilidad que experimenta la primera tendrá que tomar decisiones que no sólo las afectarán a ellas sino a todo Puerto Rico.

La universidad del Estado, conocida como la Universidad de Puerto Rico (UPR), cuenta con once recintos y hace apenas un año tenía $71,569^{1}$ estudiantes, pero a marzo de 2011 atiende sólo $62,292^{2}$. Esta reducción drástica de estudiantes en estos últimos doce meses se ha debido a un alza en los costos que un sector evidentemente mayoritario del estudiantado ha combatido y que ha afectado la institución con dos huelgas, una más duradera en la primavera del 2010, la otra mucho menos extensa y no constante en los meses de enero y febrero de 2011. Se puede suponer que algunos estudiantes, según han alegado, han dejado de estudiar por el alza en los costos. Otros se han transferido a universidades privadas, cuya matrícula, también en este último año, ha aumentado de 166,143 a 177,803³.

Las tres unidades más grandes de la UPR son el Recinto de Río Piedras, con facultades graduadas y de bachillerato universitario en ciencias naturales, ciencias sociales y estudios humanísticos, así como en arquitectura, derecho y empresas; el Recinto de Ciencias Médicas, el cual cuenta con una escuela de medicina y facultades en las diversas ramas de las ciencias de la salud, para estudiantes graduados y subgraduados; y el Recinto de Mayagüez, con múltiples programas en ingeniería y las ciencias naturales, ciencias sociales y estudios humanísticos, también a nivel graduado como sub graduado.

La UPR también tiene ocho unidades más pequeñas que no ofrecen estudios graduados, pero sí bachilleratos universitarios. Están distribuidas a través de la isla. Fueron creadas en su mayoría a finales de los años sesenta y comienzos de los setenta cuando la población estudiantil se multiplica no sólo en Puerto Rico, sino en el mundo entero. En algún momento fueron parte de lo que se llamó la Administración de los Colegios Regionales, pero con las enmiendas de 1993 a la Ley Universitaria los seis colegios que todavía estaban bajo su 
sombrilla, obtuvieron la autonomía fiscal y administrativa de la que ya gozaban las otras unidades y que ellos históricamente habían reclamado.

En los momentos en que escribo se trabaja en una nueva Ley Universitaria y uno de los asuntos que se ha sugerido que se discuta es si estas unidades deben continuar disfrutando de la autonomía que se les concedió hace ya dieciocho años o si debe crearse otra instancia administrativa en la que puedan compartir procesos de todo tipo, para que de este modo, puedan, supuestamente, economizarle recursos al estado.

El grupo de trabajo nombrado por el actual gobernador para crear un nuevo estatuto universitario cuenta entre sus miembros personas que acostumbran expresarse en los medios de comunicación, como también en los foros universitarios de los que han participado, en contra de los tradicionales reclamos de los estudiantes y los claustrales por mayor participación en la gobernación universitaria, al igual que por una mayor autonomía universitaria, reclamos comunes e históricos de la dinámica universitaria latinoamericana. El grupo de trabajo no es bajo ninguna circunstancia representativo; no ha sido avalado ni mucho menos recomendado por senados, facultades, consejos estudiantiles o el claustro completo de alguna de las once unidades del sistema. Responde, es lo que se teme, a la política anexionista $^{4}$ del actual gobierno, a sus visiones neo liberales y a su encono porque es en la Universidad de Puerto Rico donde ha encontrado mayor resistencia a sus estrategias económicas "reaganescas",

El país ha visto en estos dos años de administración anexionista cómo la resistencia de las uniones a las drásticas medidas de reducción de la burocracia en las agencias públicas, mediante despidos masivos de miles de empleados, se hizo agua, mientras que los estudiantes, con alguna, aunque tímida, ayuda de los sindicatos no docentes y docentes, confrontaban insistentemente dentro y fuera de los muros al gobierno. Rompiendo con una tradición que Puerto Rico comparte con el resto de América Latina, el gobierno le permitió a su cuerpo policial que entrara en los recintos, lo que, naturalmente, ha generado muchísima indignación. El carácter antirreformista del proceso que supuestamente se está llevando a cabo dirigido a dotar a la Universidad de Puerto Rico de un nuevo estatuto constituye un antecedente histórico en el país.

Desde luego sin admitirlo, el Gobierno se apropia de una iniciativa que no le pertenece y la desvirtúa pues todo proceso reformista de la educación, sobre todo la universitaria, 
siempre ha respondido a los reclamos que ya mencionábamos de mayor participación al interior de las instituciones y de mayor autonomía hacia el exterior. Cierto es que al final de tales procesos las legislaturas y el mandatario de turno estampan sus firmas sobre documentos que han sufrido cambios extraordinarios entre el momento en que salen de las instituciones universitarias y el día en que abandonan la casa de las leyes, perdiéndose en el trayecto importantes reivindicaciones, pero en sus comienzos parten de la efervescencia que genera la posibilidad de que estudiantes y claustrales puedan por fin alcanzar condiciones de estudio óptimas y el convencimiento de que así benefician al país. Pero en esta ocasión estamos ante una situación muy distinta.

¿Qué pretende llevar a cabo este grupo de trabajo que fue designado en medio de las contestaciones informadas que le daban los estudiantes a los aumentos de matrícula que se les imponían? ¿Acabar con las iniciativas estudiantiles y docentes? ¿Ponerle fin a la participación real de la comunidad en los senados y facultades? ¿Reducir esta participación a lo nominal, en la medida en que disminuyan los porcentajes de representatividad de ciertos sectores y se los aumenten a los administradores universitarios, regresando a modus operandi propios de los comienzos de la institución hace ya más de cien años?

Si el modo en que hasta ahora se ha manejado el aumento en el costo de la matrícula es un anticipo de lo que viene, hay razones de sobra para preocuparse, tanto en lo que respecta al ejecutivo como al legislativo. En la legislatura, por ejemplo, se aprobó recientemente, igualmente estableciendo un precedente histórico asombroso, una enmienda al reglamento estudiantil para que después de que se celebren asambleas estudiantiles, se sometan nuevamente a votación, pero en línea y vía internet, los asuntos en torno a los cuales se hayan aprobado mociones o acuerdos.

Esto se hace supuestamente para que las decisiones del cuerpo estudiantil respondan realmente al sentir de una mayoría de los estudiantes y no a los pocos estudiantes que, también supuestamente, dominan las asambleas y que permanecen hasta el final, cuando ya las mayorías agotadas se han marchado, asegurándose de este modo de que sean sus convicciones las que prevalezcan.

Sin perder de vista que es inaudito que una legislatura enmiende reglamentos estudiantiles universitarios, lo que pudo haber sido una medida dirigida a fortalecer la participación estudiantil en sus foros, porque podría contribuir a que ellos y ellas estuvieran 
mejor informados e hicieran valer su sentir en torno a asuntos que son efectivamente de su incumbencia y que, igualmente, son relevantes para el país, se sabe que responde a intereses muy distintos. Se pretende evidentemente valerse de cierto estudiantado que no sólo no participa en las deliberaciones sino que también simpatiza con las políticas del gobierno actual.

Desde luego, estos estudiantes tienen derecho a simpatizar con el gobierno y dejarlo saber, dentro y fuera de la universidad, pero deberían estar dispuestos a discutir lo que sea, frente a estudiantes que difieren de ellos, antes de proceder a votar. Lo que se ha pretendido es desautorizar las discusiones e instar a votar como bloque a favor de las posiciones que defiende el gobierno; pero lo que se logra es educar en la irresponsabilidad y, desde luego, desprestigiar toda la dinámica universitaria. Porque, ¿dónde quedan las deliberaciones, la posibilidad de convencer al que no está de acuerdo, en fin, las prácticas de una sociedad democrática entre las cuales escuchar los argumentos del contrario antes de tomar una decisión es prioritario?

Pese a lo anterior, es imprescindible no perder de vista la experiencia real de frustración y molestia de muchos estudiantes, quizás de generaciones de estudiantes, que nunca se han visto representados por el liderato tradicional del movimiento estudiantil, fundamentalmente de izquierdas. Piensan estos estudiantes que se le presta más atención a asuntos extrauniversitarios que a aquellos de textura más sencilla, pero relacionados, poco dramática y más bien prosaicamente, con mejorar las condiciones de estudio, tales como más cursos disponibles, más profesores y profesores más accesibles, mejores instalaciones sanitarias, menos estudiantes en los cursos, programas de actividades culturales más atractivos, etc. etc.

\section{II}

Quizá sea este el lugar en el que se deba aclarar lo que se podría describir como el carácter híbrido de la Universidad de Puerto Rico. El sistema universitario público de Puerto Rico está vinculado al de los EEUU por la relación política que existe entre las dos naciones; la isla fue invadida por los EEUU en el 1898 y 113 años más tarde continúa siendo una colonia, la "colonia más vieja del mundo", según se describe en un libro publicado por la Universidad de Yale en los mismos Estados Unidos ${ }^{6}$. 
Tan pronto las fuerzas armadas de los EEUU llegaron a Puerto Rico se le puso fin al sistema escolar español que, a duras penas, se había implantado en nuestra isla. Lo que ocurrió con respecto a la universidad fue muy distinto. No había universidad puertorriqueña y por lo tanto no se le tenía que cambiar su personalidad. Ella, sin embargo, se fundará cinco años más tarde, en el 1903, según ya adelantábamos, y su estructura será una de corte netamente estadounidense. Esto significará que tras comenzar como escuela normal, escuela de talante universitario para preparar maestras y maestros que en la época existía en tierras de herencia hispánica como anglosajona, pronto contaría con un primer ciclo de cuatro años, conocido como el bachillerato universitario, dos décadas más tarde con un segundo ciclo de lo que se conoce en inglés como el "Masters" y algún tiempo después con el tercer ciclo de estudios doctorales. Así se organizan las universidades en los EEUU y así se fue organizando la Universidad de Puerto Rico.

Sin embargo, contrario a lo que ocurre en los Estados Unidos con sus universidades estatales ("state universities" o "state colleges") o universidades privadas (como lo pueden ser Harvard, Yale, Stanford, Princeton, MIT o Notre Dame), desde su fundación la Universidad de Puerto Rico fue vista por sus estudiantes, sus docentes y el mismo país como una universidad nacional que se debía a la nación de Puerto Rico. Desde un principio sus debates, sus reclamos y sus proyectos no fueron sólo los de una comunidad universitaria que vivía más o menos consciente de sus condiciones circundantes, sino que fundamentalmente hacía suyos asuntos que le interesaban al país, o al revés: el pulso, más rápido en ocasiones, más lento en otras, del mismo país era asumido por la academia como un reto sobre el cual tenía que expresarse, oponerse o respaldar y hasta darle dirección si era necesario.

Aunque académicamente miraba al norte y sus programas seguían el curso de los norteamericanos, en términos administrativos sufría la tensión de periódicas confrontaciones entre gerentes que deseaban parecerse a las universidades de EEUU y claustros de profesores y cuerpos estudiantiles que hacían suyos los reclamos que se vivían en el sur, sobre todo a partir de la Reforma de Córdoba de 1918. En la Universidad de Puerto Rico se ha querido siempre más autonomía del Estado y siempre se ha buscado mayor participación democrática, llevando estos reclamos, en ciertas ocasiones, a huelgas y a nuevas leyes universitarias.

El concepto de reforma universitaria y las estrategias que le corresponden han acompañado a través de las décadas al liderato universitario puertorriqueño, tema y dinámica 
de los que no se sabe mucho en el norte, pero que se viven apasionadamente en los países latinoamericanos. Nuestros departamentos, facultades, senados académicos y otras instancias son ámbitos de discusión y participación y constituyen verdaderos foros para la deliberación frecuentemente fogosa de cuestiones nacionales y no discretos espacios de trámites burocráticos incoloros, según ocurre en las universidades de EEUU y otros países del mundo. Naturalmente, en algún momento nos tenemos que preguntar si tal tradición universitaria ha sido o no ha sido beneficiosa para nuestros pueblos.

De acuerdo al censo más reciente del gobierno de los Estados Unidos, al 2010 la isla de Puerto Rico tiene 3,725,789 habitantes $^{7}$. De éstos, casi 250,000 cursan estudios universitarios o post secundarios, lo que arroja un porcentaje alto de estudiantes universitarios per cápita. Uno de cada quince puertorriqueños se encuentra llevando a cabo estudios post secundarios. Si a esto le añadimos que el sistema escolar gubernamental atiende alrededor de medio millón de jóvenes y las escuelas privadas, religiosas y no religiosas deben estar ofreciéndole servicios a otros cien mil, aunque sobre esto no hay certidumbre pues algunos alegan que hay muchos más estudiantes en escuelas privadas, vemos que PR es una sociedad ligada de forma muy especial a la educación.

En cualquier día del año, uno de cada cuatro puertorriqueños se encuentra en alguna aula de estudios. Pero aquí tendríamos que cuestionarnos si ello nos beneficia o si la sobreabundancia de supuesta escolaridad es una mera formalidad que no se traduce en lo que el país pretende al fomentarla.

No siempre fue así. Los españoles fueron extraordinariamente tacaños con las aspiraciones puertorriqueñas de tener un sistema educativo amplio y contar con una universidad y sólo comenzamos a sentir el impacto de lo que la Ilustración había convertido en una obligación en otros lugares cuando España ya iba de salida. Contrario a lo que ocurrió en la vecina República Dominicana, en donde ya en 1538 se funda la Universidad de Santo Tomás de Aquino, y en el Perú, donde en 1551 se funda la Universidad de Lima ${ }^{8}$, según ya anticipábamos bajo la dominación española en PR no hubo universidad, si bien hubo múltiples reclamos y en ciertos momentos se ofrecieron cursos universitarios.

En lo que respecta a escuelas tampoco podemos decir que fuimos afortunados. Pese a la consabida Ordenanza de Fernando el Católico, de 1503, que ordenaba "que los niños de cada poblado, tanto los españoles como los indios, se reunieran dos veces al día en una casa

Rev. GUAL., Florianópolis, v.4, n. 1, p.82-109, jan./fev/mar/abr. 2011 
edificada al lado de la iglesia para recibir instrucción en la lectura, la escritura y en los ritos y oraciones de la Iglesia del cura párroco"9 , la educación escolar en Puerto Rico brilló por su ausencia. Siempre hubo, aparentemente, buenas intenciones y podemos leer sobre las aun mejores intenciones que destilaban algunos documentos, pero el hecho es que se nombra al primer maestro de escuela pública de Puerto Rico en el año de $1732^{10}$, casi un cuarto de milenio después de comenzada la conquista ibérica.

Nos imaginamos, según hemos dicho, que habría buenas intenciones, pero la voluntad que se necesitaba para proveer los recursos que debían corresponder a los admirables propósitos, no se veía por ningún lugar. Por ejemplo, ya desde el 1718 se contemplaba este nombramiento, pero no se había podido materializar por lo que se sospecha que era una "falta de recursos del gobierno de la capital"11. Ocurre algo similar con el "Directorio General", documento administrativo emitido por uno de los gobernadores de la isla, don Miguel de Muesas, en el 1769.

Allí "ordena abrir una escuela en cada partido para que a ella asistan la mitad, por lo menos, de los niños de cada familia". En el documento también "ordena que la escuela matricule niños blancos, pardos y morenos libres". Instituye un programa de estudios; "asigna un sueldo ... para el maestro"; establece "requisitos que deben tener los aspirantes a maestros y unas normas que deben seguir en el desempeño de sus funciones"; crea "un sistema de escuelas municipales como las que existían en San Juan ..."12. ¿Pero se cumplieron estas promesas?

De acuerdo a la información que se tiene, no se cumplió con ellas y la situación de la educación escolar no mejoraría mucho durante los próximos cien años. Entonces hacia la última tercera parte del siglo diecinueve, una serie de "decretos orgánicos" van supuestamente transformando las estructuras de instrucción en el país y el por ciento de estudiantes en escuelas va mejorando del $4.9 \%$ que había en 1864 , a $11.6 \%$ en 1878 , hasta alcanzar el $18.1 \%$ en el año de la invasión, el $1898^{13}$. Estos porcentajes, sin embargo, distan de reflejar lo que el país y su gente se merecía.

En lo que tiene que ver con las instituciones universitarias en suelo puertorriqueño es todavía mucho menos lo que se puede señalar como positivo bajo la hegemonía española. Llama la atención que a la altura de 1867 un puertorriqueño ilustrado, Manuel Corchado Juarbe, abogaba por lo que él llamaba "una Universidad literaria en la menor de las Antillas 
españolas" e insistía en que la fundación de una universidad entre nosotros no "ocasionaría algún prejuicio a la Metrópoli"14.

A través de los siglos, si los puertorriqueños aspiraban a una educación universitaria, no tenían otra alternativa que la de exiliarse. Se viajaba a Santo Domingo o a Caracas, Venezuela, según escribe Pedro Tomás de Córdoba en $1818^{15}$. Otros iban a Cuba o a España, según fueron los casos del legislador José de Diego y del educador y pensador Eugenio María de Hostos, respectivamente.

En el 1844 se gestó una iniciativa para crear una universidad en Puerto Rico, protagonizada por un académico español que por sus ideas liberales había tenido que abandonar la Universidad de Santiago en España. Rufo Manuel Fernández, profesor de Física y religioso, hizo lo posible por adelantar la idea, pero su primera e insuperable dificultad consistió en conseguir personal docente. Se optó entonces por becar a algunos jóvenes talentosos para que fueran a España a prepararse y luego regresaran a asumir las cátedras. Los dos jóvenes, por cierto, le servirían luego al país honrosamente, pero la universidad no llegó a fundarse ${ }^{16}$.

En el 1879 un grupo de farmacéuticos, abogados y médicos revivieron la idea de una universidad en la isla, ofreciéndose a enseñar gratuitamente, atendiendo de esa forma el argumento que siempre manejaba el régimen de que no había dinero para ello, pero la propuesta fue rechazada ${ }^{17}$. Luego en el 1888, las autoridades españolas aceptaron la propuesta del Ateneo Puertorriqueño de ofrecer cursos universitarios en sus instalaciones. Profesores de la Universidad de la Habana entonces vendrían a la isla a examinar a los estudiantes. El gobierno se comprometió con pagar los viajes, pero la Institución de Estudios Superiores, según se conoció y la cual generaría tanto entusiasmo, atendió 33 estudiantes el primer año, 22 el segundo, pero ya para el tercero el régimen decretó que no contaba con el dinero para sostenerla ${ }^{18}$.

Uno de nuestros primeros escritores, Manuel Alonso, quien había escrito contento ${ }^{19}$ sobre la posibilidad de que se fundara por Rufo Fernández y otros, por fin, la institución de estudios universitarios que se quería y se necesitaba, consciente más tarde de que la idea había muerto, cita a un "modesto fraile franciscano" que escribe: "Parece que sobre instrucción, en este país, pesa una maldición. ¡Cómo ha de ser! ¡Paciencia"20! 
Tras la invasión de los EEUU en 1898, la isla definitivamente se fue transformando y tanto cierta escolaridad como el posible acceso a una educación universitaria se hicieron una realidad. En 1903 se funda la Universidad de Puerto Rico (UPR) como escuela normal en un pueblo del noreste de la isla, Fajardo, pero pronto se muda al pueblo de Río Piedras, que hoy es parte de San Juan, la capital del país, y parte de lo que hoy se conoce como el área metropolitana.

En 1912 se creará la primera universidad privada, la Universidad Interamericana de Puerto Rico (UIPR), en un pueblo del suroeste de la isla, San Germán. Décadas más tarde se fundarán otras instituciones de educación superior, algunas religiosas como la UIPR, otras seculares como la UPR. Entre las primeras se deben mencionar la Pontifica Universidad Católica de Puerto Rico (PUCPR), la Universidad del Sagrado Corazón (USC) (durante décadas un colegio de niñas, luego colegio universitario exclusivamente para mujeres; hoy universidad que atiende mujeres y hombres); entre la segundas, el Sistema Universitario Ana G. Méndez (SUAGM) y la Universidad Politécnica de Puerto Rico (UPPR). La UPR, la UIPR, la PUCPR y el SUAGM tienen múltiples recintos universitarios a través de la isla.

\section{III}

Aunque Puerto Rico posee su instancia de reconocimiento y acreditación de IES, conocido como el Consejo de Educación Superior (CES), todas sus instituciones universitarias necesitan ser acreditadas por la "Middle States Commission on Higher Education", agencia de los Estados Unidos que atiende las universidades de los estados de Delaware, Maryland, New York, New Jersey, Pensylvannia, las universidades de Washington DC, Puerto Rico, Islas Vírgenes y algunas universidades del extranjero.

Esta acreditación por la "Middle States", que es como se le identifica comúnmente, es un requisito si se aspira a que los estudiantes de escasos recursos económicos puedan recibir ayuda económica del gobierno federal de los Estados Unidos ("Pell Grants"), igual que lo hacen los estudiantes norteamericanos en cualquiera de sus universidades. Un porcentaje altísimo del estudiantado puertorriqueño, tanto en instituciones privadas como en públicas, recibe esta ayuda ${ }^{21} .67 .62 \%$ del presupuesto de la universidad del Estado, la UPR, proviene de la subvención que el Estado le concede desde hace casi medio siglo ${ }^{22}$. 


\section{REALINEAMIENTO UNIVERSITARIO EN LA ISLA DE PUERTO RICO}

Los once recintos que la componen reciben el 9.66\% de los ingresos anuales al fisco, lo que implicó en años pasados un presupuesto anual de alrededor de 900 millones de dólares. El presupuesto de las universidades privadas proviene fundamentalmente de lo que pagan los estudiantes y algunas, muy pocas, actividades de recaudación de fondos. Muy naturalmente, ha habido un reclamo de estas últimas para que el gobierno local (el llamado estatal, no el federal que en nuestro caso tiene su sede en Washington, DC), contribuya también a su mantenimiento, alegando que hacen mejor uso de los fondos que administran.

La respuesta a este reclamo ha dependido, según ocurre en muchos de los temas controversiales del país, del partido que detente el poder. Los dos partidos que se han dividido la administración de Puerto Rico en décadas recientes son el autonomista Partido Popular Democrático (PPD), un partido de centro que aboga básicamente por la relación existente con los EEUU, aunque con mayor soberanía, y el anexionista Partido Nuevo Progresista (PNP), un partido conservador que aboga por hacer de Puerto Rico un estado (el 51) de los EEUU.

Entre el 1940 y el 1968, prevaleció el primero; luego tras el 1969 se han intercambiado el poder más o menos cada ocho años. La respuesta del PPD al reclamo ha sido negativa; la posición del PNP ha sido receptiva, pero la constitución de Puerto Rico no les ha permitido asignarles fondos, si bien en una de sus administraciones, mediante un programa de becas estudiantiles, lograron canalizar cantidades significativas a las universidades privadas que fueron cuestionadas en los tribunales y posteriormente anuladas cuando el PPD regresó al poder en el año 2001. De vueltas a la administración del país desde el 2009, el PNP no ha desarrollado estrategias análogas para fortalecer las universidades privadas, pero sus actuaciones hacen pensar que lo ha estado contemplando, aunque ello implique debilitar la base financiera de la UPR.

Habría que ver si el aumento del número de miembros de la Junta de Síndicos, la cual tiene a cargo el nombramiento del presidente y de los once rectores, con la participación y consejo de la comunidad universitaria, de 13 a 18 miembros no es una medida dirigida a debilitarla, mucho más efectiva que cualquier otra, inclusive que aquellas de tipo económico. Que ahora el partido de gobierno no sólo posea una mayoría, que ya la tenía, sino que cuente con una mayoría aplastante ${ }^{23}$, podría estar contribuyendo a que los puertorriqueños pierdan la fe en una institución que ha sido de un valor extraordinario para Puerto Rico en el último siglo y que, sin que esto implique rechazar o subestimar las aportaciones que han hecho y

Rev. GUAL., Florianópolis, v.4, n. 1, p.82-109, jan./fev/mar/abr. 2011 
harán también las universidades privadas, seguramente podría continuar siéndolo en el futuro.

El cinismo con que se comenta actualmente el control partidista de la Junta de Síndicos obtenido por el partido en el poder augura que el ciudadano común no la tendrá en la estima que antes la tenía y por lo tanto estará menos dispuesto a concederle y a respetarle un ámbito muy particular que es el del saber, en la medida de lo posible un ámbito desinteresado. Pero, ¿continuará ese ciudadano dispuesto a subvencionarla con las contribuciones (impuestos) que le paga al gobierno u optará por ejercer presión para que se le ponga fin al respaldo que hasta entonces recibía del Estado?

Quizás es esa misma historia de tanto valor la que lleva al anexionismo a mirarla siempre con recelo. Probablemente no desde sus mismos principios, porque en aquel momento estaba en manos de administradores que, podemos suponer, le rendían pleitesía a los funcionarios estadounidenses que representaban la posibilidad de tener una institución universitaria después de siglos de espera. Pero no pasaría una década para que se percibiera que la institución se alineaba más cómodamente con ideas y proyectos que se identificarían con la autosuficiencia del país y por lo tanto con intereses autonomistas e independentistas.

La fundación en el 1912 de un segundo recinto universitario de la UPR en la ciudad de Mayagüez, en el oeste del país, aparentemente tuvo tanto que ver con que el entonces Presidente de la Cámara de Delegados de Puerto Rico, José de Diego, quien ya era un legislador y poeta conocidísimo a través de toda la isla por la defensa de la independencia nacional, impulsara un recinto universitario para la región en que en que había nacido como con que el gobierno federal de los EEUU extendiera a Puerto Rico los beneficios de los fondos Morrill-Hatch, haciendo de la naciente institución universitaria lo que se conocía como un "Land Grant College", que como ocurría con tantos otros centros de estudios avanzados en aquel país, debería de contribuir al avance de la agricultura y la tecnología ${ }^{24}$. No es casualidad que durante muchos años la institución se conocería como el Colegio de Agricultura y Artes Mecánicas (CAAM).

El nuevo campus, según era de esperarse, se vincularía a los esfuerzos del país por desarrollarse económicamente. Sus ofrecimientos curriculares habrían de responder a los programas de desarrollo económico que trazara el gobierno puertorriqueño. No es casualidad tampoco que, según escribe uno de los primeros historiadores de nuestra educación, aquel recinto "comenzó su trabajo académico con 126 estudiantes y una Facultad de dieciocho 
miembros, ofreciendo cursos en química del azúcar, agricultura, ingeniería mecánica, ingeniería eléctrica, ingeniería civil, economía doméstica y trabajo especial” ${ }^{, 25}$. El cultivo de la caña de azúcar era entonces nuestra mayor industria y de allí saldrían quienes habrían de atenderla técnica y científicamente.

Pero no sólo porque la UPR debe ser vista como una institución comprometida con la menor dependencia económica posible, genera desconfianza, ausencia de compromiso y hasta hostilidad de parte de administraciones anexionistas. Es que, además, la institución ha sido escenario de reclamos importantes a través de la historia de Puerto Rico. La lucha por su autonomía, que renueva cada generación, los reclamos de mayor democratización, igualmente renovados cíclicamente, han corrido paralelos a los reclamos de que se atendiera el problema colonial con la salida de los EEUU de Puerto Rico y el reconocimiento de nuestra nacionalidad, y en ocasiones de sus símbolos, como fue el caso de la bandera puertorriqueña en más de una ocasión en los años treinta y cuarenta ${ }^{26}$.

A fin de cuentas, estudiantes y personal docente y no docente han reclamado desde la UPR la imprescindible puertorriqueñización de todos los ámbitos de una sociedad en la que se pretendió suplantar el español mediante el cual se enseñaba en las escuelas hasta el 1898, por el inglés de los estadounidenses que invaden en aquel año ${ }^{27}$. Por cierto, no sería hasta la elección del primer gobernador de la isla, en el 1948, cuando por fin se haga del castellano el idioma oficial del país.

No es descabellado pensar que uno de los elementos que influye en que se incluya en nuestro primer documento constitucional una visión tan generosa de nuestra educación escolar fue la conciencia que se tenía de los reclamos tradicionales del sector universitario puertorriqueño ${ }^{28}$. En la Sección 5 del Artículo 2 de la Carta de Derechos de nuestra Constitución se lee que "Habrá un sistema de instrucción pública el cual será libre y enteramente no sectario. La enseñanza será gratuita en la escuela primaria y secundaria y, hasta donde las facilidades del Estado lo permitan, se hará obligatoria para la escuela primaria".

Esta visión generosa no es compartida por la actual administración anexionista del país. Según ya hemos sugerido, se percibe en las esferas más altas, como ha ocurrido con administraciones anexionistas anteriores, un interés en debilitar las instituciones gubernamentales que se pueden identificar con los reclamos separatistas históricos con los 
que frecuentemente hacen causa común los sectores más liberales del partido que se alterna, cada ocho años, con ellos.

Naturalmente, la Universidad de Puerto Rico, que continúa siendo concebida por el anexionismo como un centro de resistencia a las estrategias políticas que desean la incorporación del país a los EEUU, es la institución más desdeñada y ciertamente objeto de estrategias que a la postre, sin embargo, serán perjudiciales para este sector ideológico. Pues, como se ha hecho evidente a través del mundo, la investigación y el desarrollo ( R \& D o Research and Development) de países que son exitosos económicamente en nuestra época están directamente vinculados a la inversión que éstos hacen en sus IES. Y desde luego, el éxito económico de estos países son proporcionales a la inversión que hacen en investigación y desarrollo. Por lo tanto, en este contexto la universidad, según se puede leer en un libro reciente en torno al tema, se ha vuelto "indispensable"29. Quien la desprecie está condenado a sufrir serias consecuencias o a prolongar su dependencia en la metrópolis, que sería el caso de Puerto Rico.

Por otro lado y según adelantábamos, como la UPR ha recibido tradicionalmente una buena tajada del presupuesto nacional de Puerto Rico, un 9.66 de los ingresos al físco, es necesario preguntar si ha hecho buen uso de estos recursos. Desde la perspectiva de las universidades privadas, se podría alegar que este monopolio de los recursos públicos, aunque hoy drásticamente reducidos, no es la mejor forma de invertir adecuadamente en la educación superior de un país en desarrollo.

¿Ha cumplido adecuadamente la UPR con lo que le correspondía llevar a cabo, de acuerdo a la Ley que la define ${ }^{30}$ ? ¿Habrá contribuido adecuadamente a "resolver democráticamente" los problemas que afectan al país? ¿O no se habrá aislado, más atenta a los intereses de su personal que al "estudio de los problemas de Puerto Rico"31? El país no ha tenido una discusión adecuada en torno a este asunto. Que sepamos sólo algunos economistas y funcionarios de las universidades privadas han planteado, más bien informalmente, que se enmiende la Ley de la UPR para que el dinero se distribuya competitivamente entre las distintas IES, privadas y públicas. Alegan que se le daría un uso más razonable de esta forma. Sea como fuere, no se ha dado la discusión informada que se debía haber tenido en torno al asunto.

Rev. GUAL., Florianópolis, v.4, n. 1, p.82-109, jan./fev/mar/abr. 2011 


\section{REALINEAMIENTO UNIVERSITARIO EN LA ISLA DE PUERTO RICO}

Un ángulo que se podría utilizar para fomentar la discusión podría ser el de las experiencias de universidades estatales norteamericanas que como la UPR han disfrutado de un ingreso anual más o menos garantizado. Se debe señalar, sin embargo, que la subvención gubernamental en los EEUU nunca ha sido tan alta como lo ha sido tradicionalmente en PR. Sea como fuere, esta subvención pública se ha reducido a través de las décadas y hoy representa un porcentaje menor en el presupuesto global de las instituciones. Sobre esto escribe el economista Waldemiro Vélez que "vale la pena destacar el mayor peso relativo que tienen las asignaciones estatales en el caso de Puerto Rico, el que ronda el 68\% de los ingresos, en comparación con ... otros estados ... En éstos, dicha asignaciones van del $10.20 \%$ del total (Wisconin) al 54.10\% (Florida).

Cabe puntualizar, además, que en este renglón el promedio para los estados Unidos (35.63\%) es prácticamente la mitad del correspondiente a Puerto Rico (67.62\%). El bajo por ciento de Wisconsin en este renglón se puede deber a que las Asignaciones Federales en dicho estado ascienden al $32.92 \%$ de los ingresos totales de las IES públicas en el año en cuestión" $^{, 32}$. Esto significa que las instituciones universitarias norteamericanas han salido a buscar financiamiento alterno y con éxito pues su dependencia en el gobierno se ha ido reduciendo. En Puerto Rico la subvención gubernamental que, según habíamos escrito, representa el $9.66 \%$ de los ingresos al fisco, constituye todavía un $67.62 \%$ del presupuesto global de la universidad pública ${ }^{33}$.

El restante $34.38 \%$ provenía en el momento en que Vélez lleva a cabo el estudio de la matrícula y cuotas que pagan los estudiantes $(8.43 \%)$, de los contratos y "grants" que obtienen los profesores para sus investigaciones del gobierno federal (18.93\%), de donaciones $(1.08 \%)$, de sus empresas $(.84 \%)$, del fondo dotal $(.90 \%)$, y otros renglones poco significativos. En los Estados Unidos el porcentaje que representa la ayuda gubernamental asignada, no competitiva, es mucho menor que en Puerto Rico y el dinero que los estudiantes pagan por su matrícula y lo que los profesores consiguen mediante "grants" es mucho mayor.

Pero esto no responde las preguntas obligadas porque sólo atiende lo que tiene que ver con una capacidad, muy importante desde luego, pero no exclusiva, que la UPR debió haber desarrollado para irse labrando un espacio de autonomía que entonces no se le estaría escamoteando hoy, lo que definitivamente hubiera sido deseable. Se trata de algo muy importante, pero no lo es todo. No se deben perder de vista otras aportaciones. 
Por ejemplo, cuántas personas ha preparado la UPR a través de los años, cuántas personas ha preparado que han hecho importantes contribuciones a la vida pública, al mundo de los negocios, a la convivencia en el país, a las ciencias y a la tecnología, ja las artes!, son interrogantes vitales. Cuánto ha influido la universidad pública a resolver los problemas acuciantes del país, cuánto ha contribuido a adelantar ella misma la economía, no a través de sus egresados, son otras preguntas muy relevantes. Se necesitaría tomar en consideración cuántas investigaciones se han llevado a cabo allí, más el valor de estas investigaciones, cuántos artículos de revistas de prestigio, los libros influyentes que han escrito sus profesores e investigadores, las invitaciones que reciben éstos para asesorar agencias gubernamentales, organizaciones privadas, cuerpos internacionales, etc. Basándose en estas respuestas, que están accesibles pues las once unidades están obligadas a someter informes anuales, es que se puede evaluar si cumplió y cumple adecuadamente con lo que le correspondía hacer.

¿Se estarán tomando en consideración estos temas en el proceso de tomar decisiones en torno a ella? Al llevar a cabo cambios académicos y administrativos en toda universidad, por no utilizar la palabra reforma, ya tan trivializada, se parte necesariamente de metas amplias y objetivos específicos. La discusión en torno a éstos es quizás la parte más importante de un proceso de transformación universitario. Luego se tiene que ser muy cuidadoso ante el reto de que las estructuras que se creen, respondan fiel y adecuadamente a lo que se quiso crear. Pero importante es saber a qué habrá de responder a partir de entonces la institución. Nos preguntamos a qué podrán responder los cambios que supuestamente se están gestando por el grupo de trabajo nombrado por el gobernador para dotar a la UPR de una nueva Ley Universitaria.

\section{IV}

La UPR ha pasado por tres transformaciones fundamentales en su historia. Su creación en 1903 respondió principalmente al proyecto estadounidense dirigido a que olvidáramos la lengua española y nos convirtiéramos en ciudadanos angloparlantes. Hasta comienzos de la década de 1920, un porcentaje altísimo de estudiantes pertenecía la Facultad de Pedagogía. Es en este contexto en el que se da su primer cambio, materializándose en la Ley Universitaria de 1925, la cual podemos decir que respondía al reclamo de que la institución por fin reconociera que estaba anclada en Puerto Rico y que tenía que responder a los intereses de los 
puertorriqueños. Aunque el primer Canciller bajo la nueva ley fue un estadounidense, ya el segundo sería un puertorriqueño y a partir de entonces siempre lo serían.

El proceso de puertorriqueñización en aquellos años veinte y luego durante los treinta no siempre fue fácil pues en ocasiones respondía a una visión de lo puertorriqueño más española que caribeña, si bien debe señalarse que su ajuste con la realidad fue mucha más ágil que la que sufrió el sistema escolar durante aquella época en la que todavía se reclamaba por las autoridades y hasta por el Presidente de los Estados Unidos que se intensificara la enseñanza "en" inglés, no sólo del inglés.

Por ejemplo, a finales de los veinte se crea en el campus principal de la UPR, el que ya se localizaba en la ciudad de Río Piedras, un Departamento de Estudios Hispánicos que no sólo contaría a través de su historia con prestigiosos investigadores puertorriqueños y extranjeros, sino que representaría ante la comunidad académica internacional el compromiso de nuestro país con la herencia ibérica ${ }^{34}$, compromiso que posteriormente sería cuestionado a medida que fueran surgiendo estudios que habrían de defender nuestra ascendencia africana ${ }^{35}$.

La segunda transformación significativa de la UPR se da a raíz de la victoria que obtiene en las elecciones (coloniales desde luego) de 1940 el recién fundado Partido Popular Democrático que se basaba en las mismas visiones populistas que parecían prevalecer en la mayoría de las repúblicas de América Latina. La Ley Universitaria de 1942 responderá a las visiones de una generación de jóvenes que no accidentalmente estaban familiarizados con los reclamos reformistas de los universitarios latinoamericanos que habían cuajado en la Reforma de Córdoba y que habrían de influir en el mismo desarrollo del populismo del área.

Se querrá entonces una universidad al servicio del desarrollo (populista) del país y se legislará para hacerlo posible. No está de más recordar que aunque la legislatura puertorriqueña era elegida democráticamente por nuestros ciudadanos, el país era regido por un gobernador nombrado personalmente por el Presidente de los Estados Unidos y sobre cuyo nombramiento los puertorriqueños no teníamos otros recursos que los morales, para expresarnos. Esto continuaría así hasta el 1947, cuando entonces se nombra un gobernador nativo, y el 1948, cuando finalmente podremos elegir nuestro primer gobernador.

La agenda de la Ley Universitaria de 1942 era evidente. La cuarta y subsiguientes oraciones de la "Declaración de Principios", que es la primera parte del documento, dicen así: "Estudiar los problemas fundamentales de Puerto Rico; Extender al pueblo los beneficios de 
la cultura; Preparar servidores públicos”. La universidad se dirigía a respaldar al gobierno en su proyecto de combatir la pobreza rampante de la época. Naturalmente, la visión de la cultura que el documento reconoce como algo que había que llevarle al "pueblo" es muy común en aquella época, hoy sin embargo objeto de muchas discusiones, pero su foco aquí y en otros aspectos es el "pueblo" y el Estado con su institución universitaria venía a socorrerlo.

El tercer cambio o reforma que vive la UPR se cristaliza en la Ley Universitaria de 1966, que es todavía, pese a algunas enmiendas, fundamentalmente la ley que está vigente. Tras años de consolidación institucional en los que se percibe que se le presta más atención a la tradición occidental que a nuestra realidad caribeña, los sesenta se presentan como una época de múltiples reclamos. Se exige un relevo del liderato político del país y del liderato universitario.

Además, se reclama una universidad que rechace el militarismo y abrace las nuevas visiones de una juventud que cree encontrar innovadores caminos para atender los viejos problemas sociales La Ley del 1966 reconocerá por fin "los valores culturales del pueblo puertorriqueño" que se tienen que "conservar, enriquecer y difundir", lo que constituye una modificación importante de la perspectiva que había dominado la reformas de los cuarenta, pero además no hace referencia a los "servidores públicos" y sí al estudiante y "su responsabilidad como servidor de la comunidad".

Aunque esta ley le da más participación a las facultades y a los estudiantes, la universidad todavía responde a lo que entonces era su Consejo de Educación Superior (CES), hoy su Junta de Síndicos. Se consulta y se le da participación para que hagan recomendaciones a la hora de designar presidente, rectores, decanos y directores, pero el peso de los nombramientos recae en las autoridades institucionales que no siempre podrán resistir las presiones del partidismo exacerbado que vive el país. No obstante, la Ley del 1966 era en términos generales, si se me permite la simplificación, más democrática y en este sentido continuaba la tradición de concederle mayor participación a la comunidad universitaria.

Los cambios que luego en el 1993 se le harían a esta ley, según ya decíamos, permitirán que dos profesores o profesoras y un estudiante sean miembros de su Junta de Síndicos, una vez más cumpliendo con una tradición de acoger afirmativamente, aunque nunca completamente, los reclamos de participación. 
No debe perderse de vista que tanto al interior de la UPR como a su exterior, como en toda institución universitaria que le hace compañía a la dinámica social de su entorno, siempre abundarán las investigaciones, estudios, informes e infinidad de sugerencias de enmiendas a la Ley y a los reglamentos. Y la mayoría de ellos planteará, cuando no exija, la necesidad de hacer más participativa la institución. Así ha sido siempre en Puerto Rico y así ha sido en los últimos dieciocho años tras los cambios a la Ley de la Universidad en el 1993.

Por ejemplo, entre el 1993 y el 1995 las Facultades del sistema elaboraron un borrador de Ley Universitaria que no fue acogido por las cámaras legislativas del país. Luego entre el 2001 y el 2004 uno de estos cuerpos legislativos, el Senado, condujo un estudio, que se titula La transformación de la educación superior en Puerto Rico ${ }^{36}$, el cual no llegó a convertirse en proyecto de ley. Más o menos para la misma época, entre el 2002 y el 2009, se llevó a cabo una revisión del Reglamento General que debía de haber elevado ante las cámaras legislativas enmiendas a la Ley de la Universidad, pero éste tampoco quedó en nada.

Como ocurre en la tradición de las universidades públicas latinoamericanas, los distintos recintos universitarios constantemente hacen sugerencias dirigidas a agilizar procedimientos y a darle mayor participación a los afectados y concernidos. Tendremos que ver lo que el grupo de trabajo nombrado por el actual gobernador anexionista nos presenta para determinar si es capaz de resistir el espíritu antirreformista con el cual se designó.

Es necesario tomar conciencia de que lo que está ocurriendo en la UPR tendrá repercusiones en lo que acostumbramos llamar las universidades privadas. Estas ya atienden alrededor del $75 \%$ de los estudiantes universitarios del país, habiendo recibido cerca de diez mil más en este último año, según escribíamos. De continuar la incertidumbre que hoy caracteriza a la universidad pública es muy posible que se establezca alguna tendencia en esta misma dirección. Las implicaciones que esto tendrá para las universidades privadas es un asunto que debe comenzarse a debatir.

\section{V}

Huelga señalar que las IES privadas tendrán que crecer para poder atender un estudiantado que se aleja de la incertidumbre de una institución que ha sufrido en estos últimos años de reiterados paros y huelgas, pero que también podría verse afectada por la inseguridad presupuestaria si la situación de decrecimiento económico por la que atraviesa el 
país no experimenta algún cambio. Según ya adelantamos, el presupuesto de la UPR se nutre de los ingresos al fisco.

Si éstos se reducen, también se reduce el presupuesto de la institución. Se trata de una situación relativamente inaudita para la UPR, pues en la historia de los últimos setenta años no se había experimentado una recesión económica como la que Puerto Rico vive en estos años.

Las universidades privadas dependen en casi su totalidad de lo que pagan sus estudiantes y tienen una plantilla claustral en su mayoría no permanente, lo que le permite confrontar los ciclos económicos más difíciles de manera ágil, aunque arriesgando calidad. Si bien sus estudiantes pagan tres y hasta cuatro veces lo que pagan los estudiantes de la UPR, las ayudas gubernamentales del gobierno federal de los EEUU (los "Pell Grants") les permiten estudiar.

La pregunta que se suscita cuando se toma conciencia de esto es la siguiente. ¿Cómo es que recibiendo igual ayuda económica del gobierno federal y proviniendo de sectores sociales con más limitaciones económicas, pueden los estudiantes de las IES privadas estudiar cuando los costos de éstas son tres y hasta cuatro veces mayores que los que tienen que asumir los estudiantes de la UPR, quienes a su vez han desarrollado una campaña de resistencia y oposición a aumentos de costos que todavía no igualan, ni se acercan, a los que confrontan los primeros?

Especulando, pues no tenemos datos a mano, nos atrevemos a conjeturar que la diferencia estriba en que el estudiante de las IES privadas casi nunca estudia lejos de su hogar, mientras que un porcentaje alto de los estudiantes de la UPR, sobre todo los de los recintos en los que la oposición a las alzas ha sido más dramática, tienden a hospedarse.

Por otro lado, el hecho de que las IES privadas subsistan casi en su totalidad de fondos provenientes de las matrículas estudiantiles y éstas en un porcentaje altísimo provengan de las ayudas (Pell Grants) que reciben del gobierno federal (Privadas sin fines de lucro (not-forprofit): $91 \%$; privadas con fines de lucro (for-profit): $93 \%$. ${ }^{37}$, ¿acaso no las convierte en universidades públicas? Pero por otro lado, ¡sin esta aportación del gobierno federal - que es gobierno también - las IES privadas puertorriqueñas se verían obligadas a cerrar todas inmediatamente!

Rev. GUAL., Florianópolis, v.4, n. 1, p.82-109, jan./fev/mar/abr. 2011 


\section{REALINEAMIENTO UNIVERSITARIO EN LA ISLA DE PUERTO RICO}

En términos generales, las IES privadas en Puerto Rico se han dedicado fundamentalmente a preparar a los estudiantes menos afortunados económicamente, especializándose en la enseñanza y perdiendo de vista, con algunas excepciones, la investigación. Pero esto debe cambiar por dos razones. Primero, con la llegada de estudiantes cuyos índices de ingreso (combinado de calificaciones y exámenes de entrada a la universidad) sean mejores, las IES que los reciban necesariamente tendrán que proveerle alternativas de investigación, según se hace cada vez más a través del mundo.

La transformación de los métodos de enseñanza que está teniendo lugar, supone una participación que va más allá de la observación y escucha pasivas de lo que el profesor hace y dice. Si el estudiante ha de acercarse al conocimiento él mismo investigando, el profesor se convertirá en algo más que un enseñante, según lo ha sido tradicionalmente, y tendrá que transformarse en un mentor que también investiga y que investiga de tal modo que puede respaldar adecuadamente las investigaciones de sus estudiantes.

Es lo que está ocurriendo en las mejores universidades del mundo, está comenzando a ocurrir en la UPR y está dándose también en algunas de las universidades privadas de Puerto Rico con la llegada de nuevas generaciones de profesores que se conciben a sí mismos como investigadores, lo que es la segunda de las razones por la que el perfil de las IES privadas debe de cambiar próximamente en esa dirección. Ahora bien, lo que hoy son excepciones, deberán de llegar a ser lo habitual y las IES privadas más alertas podrían ocupar el espacio de prestigio que hoy ocupan algunos de los recintos de la UPR, prestigio que se adjudica en Puerto Rico y fuera de Puerto Rico basándose en criterios relacionados a la calidad del estudiante que ingresa en ellas y las investigaciones y publicaciones de sus facultativos.

Se abre pues un panorama atractivo para las IES privadas de Puerto Rico. Si logran atraer a un sector importante del mejor estudiantado del país y, a la vez, porque se nutren mutuamente, si logran que sus académicos vayan convirtiéndose en facultativos que investigan, podrían transformar la dinámica universitaria puertorriqueña, auspiciando un realineamiento del capital intelectual nacional que podría tener consecuencias significativas.

Estamos pues ante una dinámica que requerirá auspiciar una discusión amplia en torno a lo universitario en Puerto Rico, discusión que por lo demás debe ser objeto de atención permanente por lo que las IES significan para todo país, pero sobre todo para países que se 
describen como en desarrollo, que es el caso de Puerto Rico. Naturalmente en Puerto Rico hay situaciones muy específicas que hacen más urgente esta discusión.

Por un lado, porque la UPR atraviesa por momentos de gran incertidumbre no sólo por los paros y huelgas que ha experimentado recientemente, sino también por la posibilidad de que le impongan una nueva ley universitaria; por el otro, porque estamos ante un posible realineamiento del panorama universitario en el cual, sobre todo algunas IES privadas, tendrían que asumir funciones que hasta ahora no habían contemplado.

Es muy importante abordar el tema del rol del intelectual en esa universidad subvencionada por el Estado que está siendo afectada de manera dramática por las huelgas, los paros y las determinaciones de un gobierno que parece no creer en su universidad nacional. Es una discusión que se está dando ${ }^{38}$, pero que tiene que incluir al académico que habita las IES privadas. Tampoco debe excluir a los investigadores de las ciencias naturales y las condiciones que ellas y ellos necesitan, en las públicas y en las privadas, para desarrollar sus investigaciones de naturaleza tan distinta a la de los que nos dedicamos a las disciplinas socio-humanísticas.

Es preciso estudiar lo que significa para el estudioso de nuestra época dejar de ser subvencionado por el Estado y trabajar para IES privadas con sus misiones y metas específicas. Aunque en lo que respecta a este asunto se debería partir del hecho de que ya, hace algún tiempo, una mayoría de los estudiosos del país trabaja para IES privadas.

Claro que el tema del nuevo rol que podrían comenzar a desempeñar las IES privadas en el panorama nacional de una sociedad como la nuestra es quizás el que más urgencia tenga. Si llega a ocurrir, según lo hemos sugerido, que la universidad subvencionada por el Estado se continúe debilitando, bien porque su presupuesto se vea reducido o porque los paros y las huelgas hagan muy difícil la investigación y el aprendizaje, ¿cómo reaccionarían ellas?

¿Estarían algunas listas para subvencionar la investigación que dejaría de darse en la UPR y sobre la cual se tienen tantas esperanzas de que contribuya al desarrollo de la economía nacional? ¿Estarían algunas dispuestas a reenfocar su misión y metas para llenar el vacío que se podría crear a raíz de la situación que hemos estado describiendo.

Sea como fuera, algunas IES puertorriqueñas privadas tendrán que comenzar a prestarle mayor atención a la investigación si se aspira a un desarrollo económico que por lo menos mantenga los niveles de calidad de vida que han caracterizado al país hasta ahora. La 
industria farmacéutica, que contribuía con un porcentaje significativo al producto nacional bruto, se continúa debilitando y se teme que llegue a representar un porcentaje exiguo.

En tal coyuntura, por ejemplo, la investigación y el desarrollo (R\&D) del país necesitaría iniciativas investigativas que pudieran generar inversiones que substituyeran la producción de fármacos. Las universidades, todas, no sólo la UPR, serían los lugares donde éstas deberían realizarse.

Hay desde luego muchos temas que se han estudiado en otros lugares y que están por estudiarse adecuadamente sobre las IES puertorriqueñas. ¿Por qué no discutir, por ejemplo, la suplantación de la realidad real por la realidad virtual que se está dando en nuestras mismas IES y cómo esto afecta los procesos de aprendizaje ${ }^{39}$ ? $\mathrm{O}$, ¿cómo se transforma la universidad en la medida en que es habitada por una población más madura que la que acostumbraba poblarla hace algunos años?

En un libro reciente sobre las universidades de EEUU se nos informa que allí la edad promedio de los estudiantes ha aumentado. La universidad que acogía estudiantes entre 18 y 22 años desaparece. Hoy en día sólo $16 \%$ de los estudiantes caen en esta categoría; la mayoría tiene más de 22 años. Además, $82 \%$ trabaja y $32 \%$ trabaja a tiempo completo ${ }^{40}$. O, ¿cómo atender la proliferación de universidades del exterior en línea que no auspician la investigación y cuando la auspician no se preocupan porque esté ligada a la realidad del país? Igualmente, ¿qué pensar de las IES que son tratadas cada vez más por el gobierno y los sectores de inversión financiera como instancias reproductoras de las dinámicas económicas prevalecientes? ¿Podrán las IES producir la crítica imprescindible que tal contexto exige, sobre todo las IES privadas? ¿Pero ha provisto "la universidad” esa crítica a través de los tiempos o esto ha sido tan sólo un espejismo?

Las IES puertorriqueñas confrontan grandes retos. Los próximos años nos dirán si están a la altura de ellos o si irán perdiendo fuerza. La UPR tendrá que ver cómo continúa desarrollando bajo gobiernos que históricamente no simpatizan con sus gestiones críticas y presupuestos menores. Las IES privadas tendrán que decidir si ante la situación de alineamiento que hemos intentado describir están dispuestas a auspiciar las investigaciones que el país necesita para desarrollar la calidad de vida que sus ciudadanos se merecen. Sea como fuera, nos esperan tiempos interesantes. 


\section{REFERENCIAS}

1 Ver artículo de El nuevo día del domingo 20 de febrero de 2011, pp. 8-11, “Transformación sin protestas", sobre las universidades privadas de Puerto Rico.

2 Ver El nuevo día, p. 5, del jueves 10 de marzo de 2011.

3 Ver el artículo de El nuevo día, previamente citado, del domingo 20 de febrero de 2011.

4 El anexionismo en Puerto Rico está constituido por aquellos que buscan convertirnos en el estado 51 de los Estados Unidos.

5 El gobernador, Lic. Luis Fortuño, se expresa comúnmente como lo hacía el fenecido presidente de los Estados Unidos, Ronald Reagan, señalando que el problema que tiene el país es su gobierno y que es preferible un dólar en el bolsillo del contribuyente que en las arcas del Estado, naturalmente dando a entender que los servicios que el gobierno le ofrece tradicionalmente a las clases menos agraciadas no son tan importantes como el enriquecimiento de los individuos, de algunos individuos vale decir. Sobre su "reaganismo" ver Claridad, San Juan, Puerto Rico, 7 al 13 de abril de 2011, p. 5.

6 Trías Monge, José, The Trials of the Oldest Colony in the World, New Haven and London: Yale University Press, 1997.

7 Estos son puertorriqueños que viven en la isla. Hay ya un poco más de cuatro millones de puertorriqueños que habitan en los EEUU. Cfr. tendenciaspr.com en la internet.

8 Llamada así entre el 1551 y el 1821.

9 López Yustos, Alfono, Historia documental de la educación en Puerto Rico, 3ra ${ }^{\mathrm{a}}$. edición, Hato Rey: Publicaciones Puertorriqueñas, p. 2.

10 Ibid., pp. 22 y 23.

11 Ibid.

12 Ibid., pp. 30-32.

Rev. GUAL., Florianópolis, v.4, n. 1, p.82-109, jan./fev/mar/abr. 2011 
13 Ibid., p. 62.

14 Ver Corchado, Juarbe, M., Obras Completas (Tomo II), san Juan: Instituto de Cultura Puertorriquena, 1975, pp. 30-36 y Aragunde, Rafael, Sobre lo universitario y la Universidad de Puerto Rico, Hato Rey: Publicaciones Puertorriqueñas, 1996, pp. 1,2.

15 Osuna, Juan J., A History of Education in Puerto Rico, 2nd. edition, Río Piedras: Editorial UPR, 1949, p. 103.

16 Ibid., pp. 108 y 109.

17 Ibid., p. 111.

18 Ibid., pp. 11 y 112.

19 Alonso, Manuel, El gíbaro, cuadro de costumbres de la isla de Puerto Rico, San Juan:

Academia Puertorriqueña de la Lengua Española, Editorial Plaza Mayor, 2007, p. 192. La primera edición de El Gíbaro es del 1849.

20 Ibid., p. 282

21 Entre el $91 \%$ y el $93 \%$ en las IES públicas y un $72 \%$ en las IES privadas. Bases de datos del "National Center for Education Statistics" (NCES) del Departamento de Educación de los Estados Unidos (USDE), sobre la cantidad de estudiantes puertorriqueños que participó del "Federal Grant Aid" para el año fiscal (FY) 2008.

22 Vélez Cardona, Waldemiro, El financiamiento de la educación superior en Puerto Rico, San Juan: Ediciones Educación Superior, Gráfica Metropolitana, 2002, p. 155.

23 Entre los 18 hay dos profesores y un estudiante, electos anualmente por sus sectores. Los nombramientos de los otros miembros son por cinco años.

24 Osuna, J.J., Op. cit., pp. 250 y 251.

25 Ibid., pp. 251 y 252.

Rev. GUAL., Florianópolis, v.4, n. 1, p.82-109, jan./fev/mar/abr. 2011 
26 Ver Picó, Isabel, “Los orígenes del movimiento estudiantil universitario: 1903-1930”, Revista de Ciencias Sociales (Universidad de Puerto Rico), Vol. XXIV, nums. 1 y 2, Enero-junio 1985 e Picó, Isabel, "Los estudiantes universitarios en la década del treinta: del nacionalismo cultural al nacionalismo político", Revista de Ciencias Sociales (Universidad de Puerto Rico), Vol. XXIV, nums. 3 y 4, Julio-diciembre 1985.

27 Ver Negrón de Montilla, Aida, Americanization in Puerto Rico and the Public School System, 1900-1930, 2da. ed., Barcelona: Editorial UPR, 1975.

28 Se debe aclarar que este documento ha estado sujeto a mucha discusión desde su creación por el alegato que hacen algunos constitucionalistas de que no se trató de un proceso soberano, sino que se subordinó y todavía estaría subordinado al Congreso de los Estados Unidos.

29 Trani, E., \& Holsworth, R., The Indispensable University, Higher education, Economic Development, and the Knowledge Economy, USA: American Council on Education and Rowman \& Littlefield Publishers, Inc., 2010.

30 Ver Ley número 1 del 20 de enero de 1966, según enmendada.

31 Esta frase entre comillas, como la de la oración anterior, es una cita de la Ley número 1 del 20 de enero de 1966, según enmendada.

32 Vélez Cardona, Waldemiro, Op. cit., p. 155.

33 Ibid. Estos porcentajes y estadísticas provienen del previamente citado libro.

34 Hay que decir que a veces en perjuicio de nuestras otras dos herencias, la indígena y la africana, que no serían rescatadas adecuadamente por investigaciones y publicaciones hasta décadas más tarde.

35 Ver de José Luis González, El país de cuatro pisos y otros ensayos, Río Piedras: Editorial Huracán, 1980. 
36 Ver La transformación de la educación superior en Puerto Rico, sin lugar: Senado de Puerto Rico, Comisión de Educación, Ciencia y Política, 19 de septiembre de 2001.

37 Ver nota número 21. Se trata de datos del "National Center for Education Statistics" (NCES) del Departamento de Educación de los Estados Unidos (USDE).

38 Ver revista en línea 80grados.net

39 Digo proceso de aprendizaje a propósito pues estamos obligados a pensar más en cómo es que aprenden los estudiantes en la medida en que entra en crisis el sujeto magisterial precisamente con el acceso que en esta época tiene cualquiera a todo tipo de información.

40 Ver Taylor, M., Crisis on Campus, New York: Alfred Knopf, 2010, pp. 172 ss. 


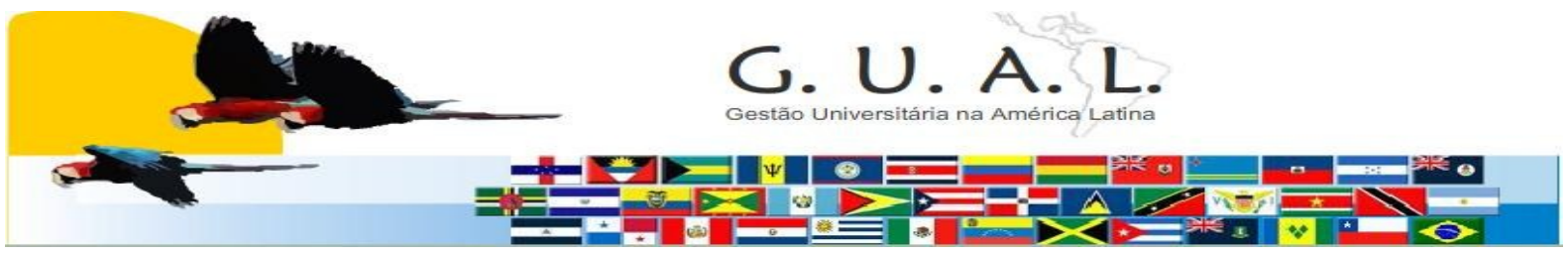

ISSN 1983-4535

\title{
REALIGNMENT UNIVERSITY ON THE ISLAND OF PUERTO RICO
}

\author{
Rafael Aragunde, Doctor \\ Universidad Interamericana de Puerto Rico \\ aragunde@yahoo.com
}

\begin{abstract}
Puerto Rico's higher education is going through a very special process. The University of Puerto Rico, which is the state university system, has recently experienced strikes and work stoppages due to a tuition increase which has been rejected by its students. In the meantime, the Governor has named a citizens commission that should work on a new University Law that will probably do away with students and Faculty rights to participate in its most important processes. Puerto Rico's experience with educational institutions, starting with the Spanish "conquista", followed by the 1898 US invasion, and culminating with the economical crises that the country has had to confront in this early twenty first century, has to be revisited so that one can understand how the island-country has had to struggle to achieve the high percentages of students registered in both schools and colleges, something that could definitely change if the pro statehood party in power gets to impose its very inclusive and neo liberal ideas on education. Yet the new situation could offer private colleges and universities, which already have a 70\% share of the country's higher education students, a chance to develop. If they decide to support R \& D and do more than simply teach its students, it will definitely contribute to a university realignment that will change Puerto Rico's higher education's personality.
\end{abstract}

Keywords: Realignment. Puerto Rico. University. 